\title{
Extralobar pulmonary sequestration as a cause of recurrent pulmonary hemorrhage in a neonate
}

\author{
Arzu Akdag, M.D. ${ }^{a}$, Sule Arici, M.D. ${ }^{b}$, Mete Kaya, M.D. ${ }^{c}$, and Ahmet Turgut, M.D. ${ }^{d}$
}

\begin{abstract}
Pulmonary sequestration is acystic or solid congenital lung malformation comprised of non functional lung tissue that does not communicate with the normal tracheobronchial tree and has a systemic arterial blood supply. There aretwo forms of sequestration: intralobar and extralobar. Its treatment is surgical resection. Here we presented a case of premature neonate with extralobar pulmonary sequestration who had respiratory failure and recurrent pulmonary hemorrhage. Following surgery, the patient showed significant clinical improvement.

Key words: pulmonary diseases, hemorrhage, pulmonary sequestration, extralobar, newborn.
\end{abstract}

http:/ /dx.doi.org/10.5546/aap.2016.eng.e21

\section{INTRODUCTION}

Pulmonary sequestration (PS) is a congenital lesion that consists of anomalous lung parenchyma that has own arterial feeding and not connected to the normal bronchial tree. PS mainly occurs in two types: intralobar and extralobar. Intralobar pulmonary sequestrations (ILPS) are masses of lung parenchyma that are contiguous with the adjacent normal lung. In contrast, extralobar pulmonary sequestrations (ELPS) are masses of lung parenchyma that have a distinct pleural covering maintaining complete anatomical separation of the mass from adjacent normal lung tissue. ELPS can be located at mediastinum or subphrenic space. ${ }^{1,2}$

ELPS is mostly asymptomatic. Symptomatic PS is usually an intralobar type presenting as infection, hemoptysis and hemothorax caused by communication to the airway. ${ }^{3}$

\footnotetext{
a Division of Neonatology.

b Department of Pediatrics.

c Department of Pediatric Surgery.

d Department of Pediatrics.

Bursa SevketYilmaz Training and Research Hospital,

Bursa, Turkey.
}

E-mail Address:

Arzu Akdag, M.D: arzuakdag@hotmail.com

Funding: None.

Conflict of interest: None.

Received: 7-15-2015

Accepted: 8-31-2015
In this paper we presented a case of premature neonate with ELPS who had respiratory failure and recurrent pulmonary hemorrhage. After surgery, the patient showed significant clinical improvement. To our knowledge, this is the first report of a newborn with ELPS complicated by recurrent pulmonary hemorrhage.

\section{CASE}

A $2100 \mathrm{~g}$ male neonate was born to a 42-yearold mother (gravida 6, parity 6) at gestational age of 32 weeks by caesarean delivery due to preterm labor and transverse presentation. He was admitted to neonatal intensive care unit because of severe respiratory distress. The mother's past and family history were uneventful. On physical examination, he was cyanotic, neonatal reflexes were depressed; spontaneous breathing was defective with nasal flaring and intercostal retraction. Other system examinations were normal. He was intubated and connected to mechanical ventilation. Surfactant was administered via endotracheal tube since the chest $X$-ray revealed reticulogranular appearance (Figure 1). On postnatal day 3 , pulmonary hemorrhage developed. There were no thrombocytopenia, coagulopathy, or patent ductus arteriosus, all of which could contribute to pulmonary hemorrhage.

Initially it was considered that pulmonary hemorrhage was caused by respiratory distress syndrome and prematurity. Despite multiple fresh frozen plasma transfusions and vitamin $\mathrm{K}$ injections, repeated episodes of pulmonary hemorrhage occurred until postnatal day 21. For detailed investigation, thoracic computarised tomography was performed. It was detected that there was an ELPS in the lower lobe of the left lung and its vascular blood supply was coming from the descending thoracic aorta (Figure 2).

The patient was operated for resection of the lesion via a standard left posterolateral thoracotomy incision. Intraoperatively, a nonventilated pulmonary parenchyma at the posterior basal region of the left thorax was noted, which was fed by two anomalous arteries arising from thoracic aorta, and directly drained into the azygos vein. (Figure 3). Complete resection 
was performed. Postoperative course was uneventful. Following surgery, significant clinical improvement was observed. The patient was extubated on postoperative day 3 and discharged on postoperative 10th day after full recovery. The patient remained asymptomatic during the follow up period of 6 months.

\section{DISCUSSION}

Pulmonary hemorrhage is a life-threatening condition that associated with many predisposing factors, such as low gestational age, presence of respiratory distress syndrome, patent ductusarteriosus, hypoxia, severe hypothermia, and congenital heart disease. ${ }^{4}$ It was suggested that defective coagulation function could only serve to exacerbate or prolong the hemorrhage rather than initiate it. ${ }^{5}$ The evaluation of these patients include a complete blood count, coagulation studies as well as chest radiograph, echocardiogram, and if necessary, broncoscopy and angiography of the bronchial arteries. Treatment options after acute deterioration required airway control and patient stabilisation focus on etiology.

PS is a cystic or solid congenital lesion consists of anomalous lung parenchyma which is not connected to the normal bronchial tree with its own arterial feeding. It was first described in 1861 by Rokitansky and Rektorik. ${ }^{2}$ There is no consensus regarding the etiology of PS, but there are two possible hypotheses: formation and caudal migration of a supernumerary pulmonary

\section{FIgURE 1. Chest X-ray revealed reticulogranular appearance}

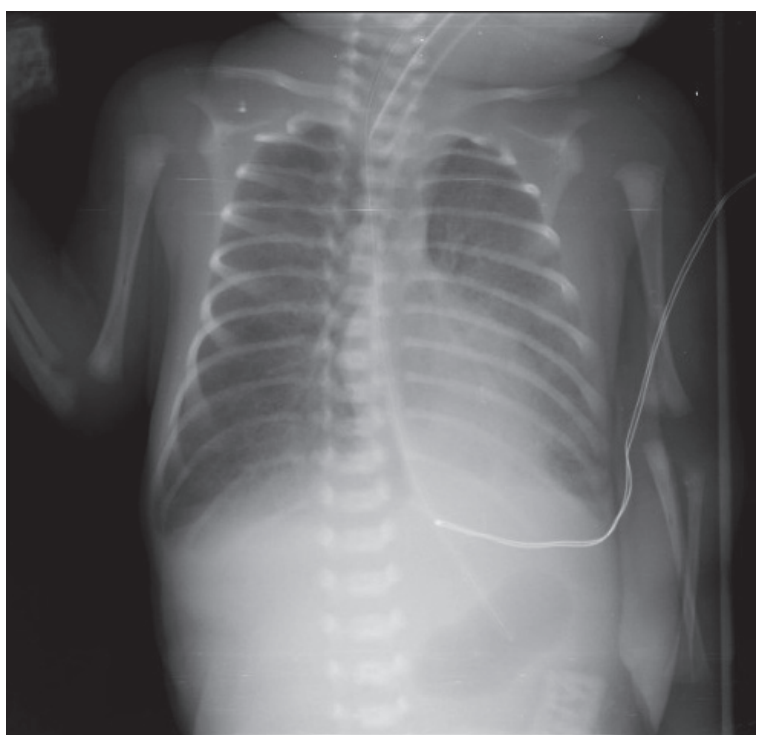

bud that accompanies the esophagus and primary pulmonary vascular deficiency, which would explain the persistence of systemic collateral circulation. ${ }^{1,6}$ PS accounts for $0.15 \%$ to $6 \%$ of all congenital pulmonary malfunctions. These lesions are divided into two types on the basis of their pleural coverings. ELPS are masses of lung parenchyma that have a distinct pleural covering maintaining complete anatomical separation of the mass from adjacent normal lung tissue. In contrast, ILPS are masses of lung parenchyma that are contiguous with the adjacent normal

FIGURE 2. Computarised tomography of the chest showing ELPS in the lower lobe of the left

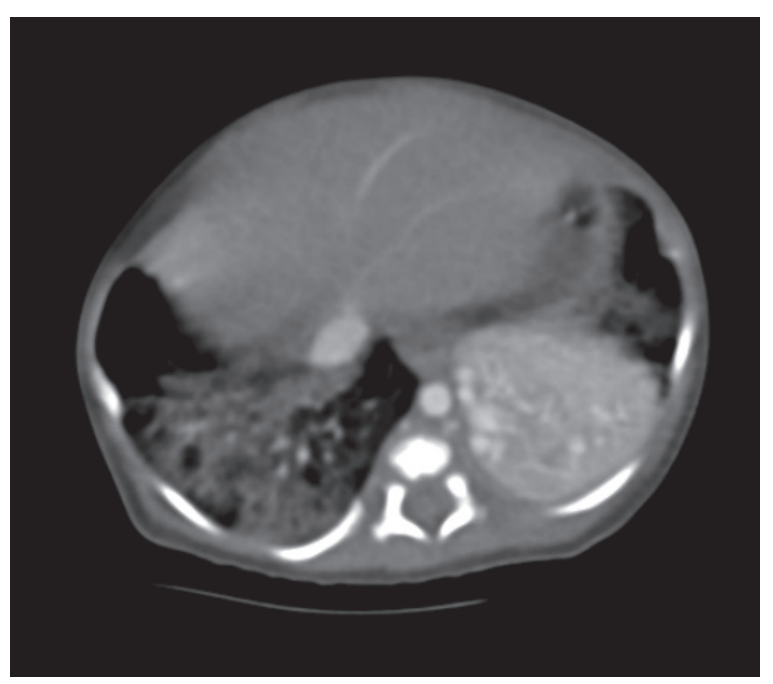

FIGURE 3. Non ventilated pulmonary parenchyma at the posterior basal region of the left thorax

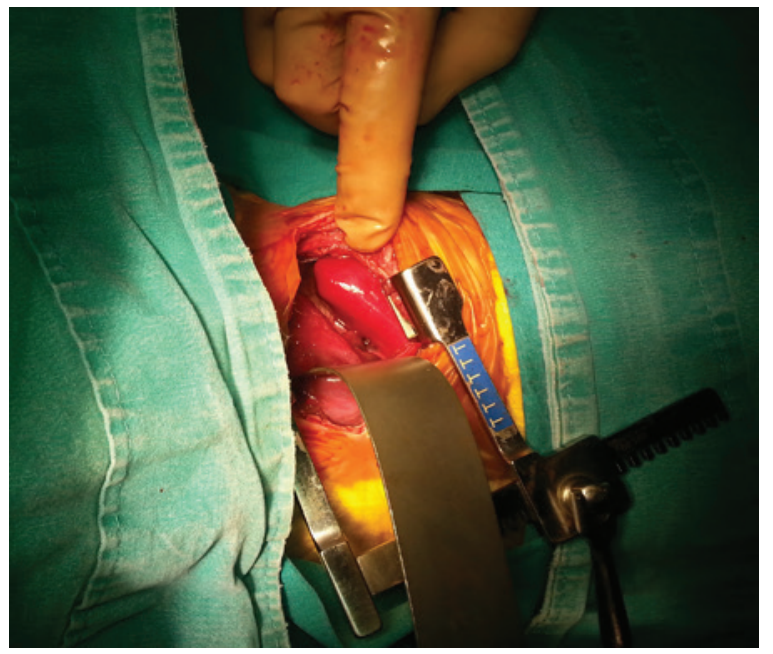


lung. ILPS accounts for $75 \%$ of sequestrations and ELPS accounts for $25 \%{ }^{1,3}$

Both of ELPS and ILPS are most frequently located in the left lower lobe. ELPS is associated with congenital deformity in which congenital heart disease, diaphragmatic hernia, hydrops, pectus e cavatum, vertebral anomalia more than ILPS. ${ }^{1,3}$ In our case, ELPS was detected in the lower lobe of the left lung without additional anomalies.

Both, ELPS and ILPS, have their own vascular blood supply usually arises from the aorta or from one of its branches. In ILPS, the venous drainage is usually into the pulmonary veins, although the veins drain through the azygos, hemiazygos or portal system in ELPS. ${ }^{1,7}$ In our case, the lesion's vascular blood supply was coming from the descendent thoracic aorta and the veins drainedinto the azygos.

The clinical manifestations of PS are fever, cough, expectoration, respiratory distress, cyanosis and hemoptysis. Although PS is mostly asymptomatic in the neonatal period, severe respiratory distress syndrome, congestive heart failure, hydrothorax, pneumonia may happen in infancy. ${ }^{1-3}$ In our case, the clinical findings were pulmonary hemorrhage and respiratory failure developed shortly after birth.

Rarely, PS may lead to pulmonary hemorrhage and hemoptysis. Although the exact mechanism is not known, it is hypothesized that vascular degeneration caused by lung compression of PS might be responsible from pulmonary hemorrhage. ${ }^{8}$ In the presented case of ELPS, the lesion had compressed the pulmonary vasculature that resulted in pulmonary hemorrhage.

In PS, treatment modalities depend upon whether the patient has respiratory distress, recurrent infections, or is asymptomatic. Treatment is controversial in asymptomatic patients or patients with incidentally detected PS. Resection is usually recommended for ILPS to prevent complications, but the optimal timing of surgery has not been established. On the other hand, in symptomatic patients, surgical excision of the lesion is recommended. ILPS usually treated with lobectomy or segmental resection, whereas ELPS can be resected by simple excision because the lesion has its own pleura and blood supply. But, the arterial supply of ELPS may arise from the subdiaphragmatic aorta, and careful identification of the vessels is important. Alternatively the patients with PS can be managed by thoracoscopy or interventional radiology. Advancing techniques in fetal surgery have made antenatal intervention a valuable option in selected cases of PS. ${ }^{8-10}$ In our case, the lesion was successfully resected via open thoracotomy.

To conclude, PS may lead to recurrent pulmonary hemorrhage in neonatal period. PS should be considered when recurrent pulmonary hemorrhage cannot be differentiated throughnoninvasive examination. A ortagraphy, Doppler angiography and MR angiography may be used in suspected patients. Computerized tomography imaging can also provide valuable information and surgical excision of the lesion would be lifesaving in such cases.

\section{REFERENCES}

1. Pires CR, Czapkowski A, Araujo Júnior E, Zanforlin Filho SM. Diagnosis of Intra-abdominal extralobar pulmonary sequestration by means of ultrasound in a neonate. Case Rep Pediatr 2013;2013:623102.

2. Tetsuka K, Endo S, Kanai Y, Yamamoto S. Extralobar pulmonary sequestration presenting as hemothorax. Interact Cardiovasc Thorac Surg 2009;9(3):547-8.

3. Ou J, Lei X, Fu Z, Huang Y, et al. Pulmonary sequestration in children: a clinical analysis of 48 cases. Int J Clin Exp Med 2014;7(5):1355-65.

4. Raju TN, Langenberg P. Pulmonary hemorrhage and exogenous surfactant therapy: a metaanalysis. J Pediatr 1993;123(4):603-10.

5. Cole VA, Normand IC, Reynolds EO, Rivers RP. Pathogenesis of hemorrhagic pulmonary edema and massive pulmonary hemorrhage in the newborn. Pediatrics 1973;51(2):175-87.

6. Morin L, Crombleholme TM, D'Alton ME. Prenatal diagnosis and management of fetal thoracic lesions. Semin Perinatol 1994;18(3):228-53.

7. Cakir U, Kahvecioglu D, Alan S, Yildiz D, et al. Extralobar pulmonary sequestration requiring intrauterine thoracentesis. APSP J Case Rep 2015;6(1):3.

8. Reynolds M. Congenital lesions of the lung. In: Shields TW, LoCicero J, Ponn RP, eds. General thoracic surgery. 5th ed. Philadelphia: Lippincott Williams \& Wilkins; 2000. Pags.937-52.

9. Laberge JM, Puligandla P, Flageole H. Asymptomatic congenital lung malformations. Semin Pediatr Surg 2005;14(1):16-33.

10. Lee BS, Kim JT,Kim EA, Kim KS, et al. Neonatal pulmonary sequestration: clinical experience with transumbilical arterial embolization. Pediatr Pulmonol 2008;43(4):404-13. 